\title{
3D DETECTION AND RECONSTRUCTION EXPERIMENTS IN RIVER BASINS.
}

\author{
C. Palestini, A. Basso ${ }^{\text {a }}$ \\ a Department of Architecture, University of the Adriatic Studies “G. D’Annunzio” Pescara, Italy \\ caterinapalestini@libero.it - alessandro.basso77@gmail.com
}

\section{Commission II}

KEY WORDS: Photogrammetry, Google Earth, 3d Reconfiguration, Digital terrain Model, Drone.

\begin{abstract}
:
The survey and representation of river landscapes require complex operations related to the habitat identifying these environments that show heterogeneous connotations in which the anthropogenic contaminations, alternating with the peripheral areas, industrial or abandoned, are united in a territory with hybrid characteristics.

In this regard it is proposed as a case study the experimentation carried out on the Pescara river basin that specifically compares two low cost detection systems comparing the results obtained to carry out environmental readings and 3D reconstructions of the territory.

The operations of analysis and data acquisition include integrated procedures used with the aim of obtaining high-density 3D reconstructions of details applied on the river portions of Pescara-Aterno. Starting from the delta towards the inside, identification samples were analyzed to focus attention on the different criticalities. Integrated methodologies were employed involving the experimental use of specific software to obtain depth maps and territorial models compatible with real ones, crossing free open shared data obtained from the Google Earth Pro, photogrammetric reconstructions and information provided on site by RPAS instruments (Remoted Piloted Air System) that use the image based methodologies to carry out an in-depth scan of the territory.
\end{abstract}

\section{INTRODUCTION}

The contribution presents an experiment that adopts an alternative executive pipeline through which photo modelling operations are carried out, captured on $3 \mathrm{~d}$ models elaborated by the popular platform of Google Earth Pro (Figure1). This procedure, highly low-cost, allows to quickly obtain a first model of semi-detailed study to be used as a basis for manual and retopological remodeling operations, for the creation of $3 \mathrm{~d}$ territorial models that can be implemented with architectural models suitable for virtual tours.

The possible loss of precision, due to the repetition of a manual photogrammetric scan on a model already generated through the same procedures, for this repository of a previous percentage of error, was supported by a verification phase after the generation of this model, in which they were compared the point clouds generated by photogrammetry (using Pix $4 \mathrm{~d}$ or Photoscan) on the Google model and the point cloud obtained from calculations on the model with direct photo acquisition through drone RPAS system. Specifically, a consumer type drone with easy portability was used, internally equipped with a GPS system fundamental for a geolocation of the resulting threedimensional model. The experimental application of this methodology based on cross-checks has allowed the extrapolation of three-dimensional plastic data and color information derived from Google Earth Pro, impossible to export directly to other platforms (as owned by Google).

The digital territorial model obtained from image-based survey procedures is then retopologized and optimized at the polygonal mesh level on Pixologic Zbrush software, an advanced sculpting program fundamental in the architectural field for a corrective intervention of the model resulting from photogrammetric selfmodelling. The three-dimensional axis can thus be implemented with further detailed models, such as parametric vegetation,

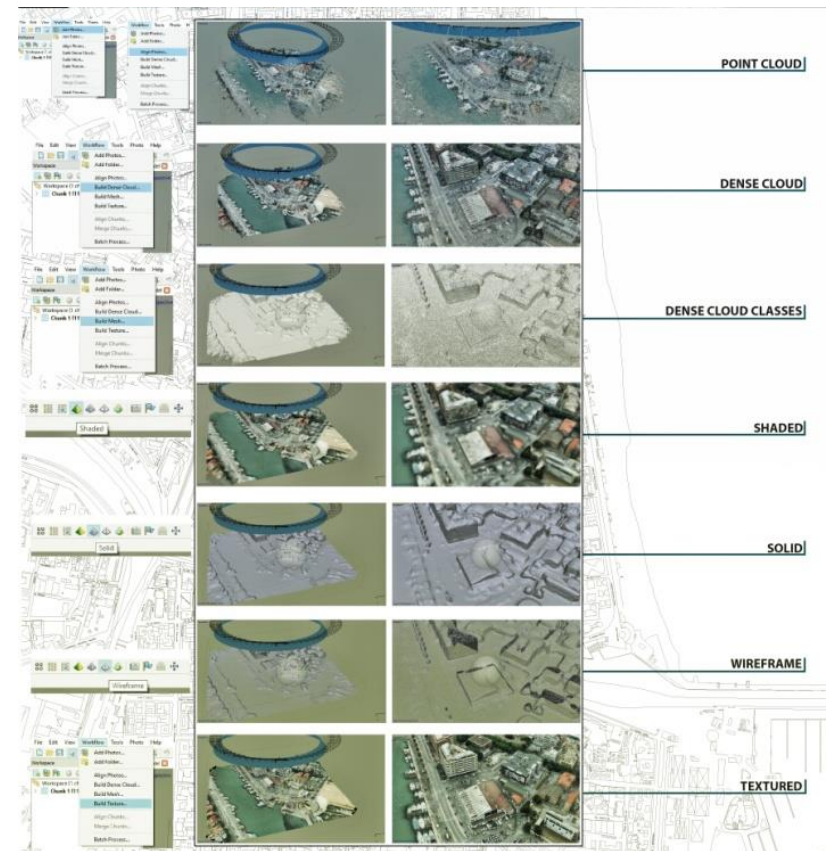

Figure 1: photogrammetric survey steps done with Photoscan by acquiring data from Google Earth with photocentric camera shots.

textures and animated shaders, compatible to be imported on current virtual platforms equipped with advanced rendering engines, as Unreal Engine 4.20 or Unity, useful to obtain visual effects of photorealism and a three-dimensional interactive exploration (Cummings and Bailenson, 2016). 


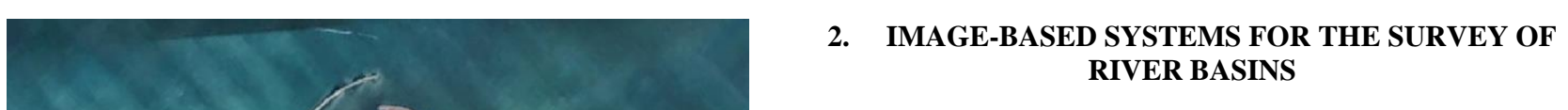

Following an experimental and low-cost approach, the case study focused on the integrated use of the free open shared data obtained from the photogrammetric reconstructions of Google Earth Pro, subsequently verified through sample comparisons with some image-based surveys carried out on the place on the same areas of analysis, in order to obtain the corresponding digital spatial models. The multinational Google, a real power in the digital industry in terms of investment, marketing and communication capacity, has focused globally on the most innovative Remote Sensing techniques, spending a lot of effort on solutions not specifically related to the Geomatic model, resulting in a sort of creative accelerator for diversified operations, from that of the territorial survey, to architectural design but also to the use of data for scientific or research purposes. Google Earth, born as an online tool to support a web directory based on a playful and attractive setting, quickly becomes a geographical tool with GIS ambitions, so as to be used by companies and organizations that use geographic data within their projects. The contemporary approach connected to the dynamics of web 2.0 aims at the development of information containers with direct creation and management of the user, thus influencing the majority of GIS technology players (Autodesk, Bentley Systems, Intergraph, ESRI, Bing 3d, etc.) to follow the navigation interface and the visual concept of Google Earth and Maps, thanks to its strong interactivity, between user and three-dimensional digital element, and its easy use. The restyling that Google has been doing since some years, implementing interesting graphic updates that make it even more useful to consult the app, allows for the first time the use of high detail self-constructive photogrammetry used in a project of global dimensions. Taking advantage of special compact planes, each with 5 cameras with wide-angle lens, placed on the four cardinal points and on the view in a normal position perpendicular to the ground floor. The $3 \mathrm{~d}$ photogrammetric scanning project acquires data by following survey paths similar to those produced with stereoscopic aerial photogrammetry, taken from a metric camera, with regular shots, functional to have an overlap of about $80 \%$ with each other. The photographic data are then optimized and photos retouched by the Google staff, partly using automatic graphic procedures, in order to normalize the chromatic aspect, the digital disturbance and any artifacts, in order to homogenize the photographic data. These procedures allow to reduce possible mesh deformation errors in the following phases of selfmodelling. The new systems of territorial survey have thus allowed Google to make available detailed models completely three-dimensional of a large number of urban centers worldwide, overlapping the detailed photogrammetric model to the previous one, obtained using only DEM data and maps generated by satellite images. This leap in quality has allowed an extraordinary database of visual information together with the possibility to better perceive at a three-dimensional level the structure of the areas analyzed, identifying the height factor of each reconfigured element and its proportional ratio between solids and voids, but also, since the models are inclusive of textures with a rather high resolution, clearly distinguishing natural elements, such as waterways and greenery, from anthropogenic ones.

The case study, considering the above mentioned background, carries out experimental operations of analysis and data acquisition on some river portions of the Pescara-Aterno, using as preliminary data the images taken from Google Earth pro, to which are added subsequent operations of verification and comparison on some sample portions (Figure 2). The procedure 
consists in the virtual simulation of drone flight, proposed with the aim of extracting the image data necessary for the subsequent phases of photomodelling, and is based on a virtual tour that follows a predetermined path, chessboard or photocentric, with an inclined shot between $30^{\circ}$ and $45^{\circ}$, within overlapping macro areas. Assuming to drive a virtual drone, imagining to follow the path of the planes of Google through a precise subdivision into submodules, we identify precise nodal points around which to take shots and thus collect complete three-dimensional data, both on the geomorphological conformation of the ground, both regarding the layout and height of the built (Soler et al., 2017). The numerous frames obtained from the virtual tour movie were then processed in Agisoft Photoscan, in order to obtain, through a procedure of alignment of the homologous pixels, a dense points cloud from which was generated, using the same points as vertices, a triangular polygonal mesh of the entire area, on average detailed enough to trace the plastic connotations similar to the reference model.

\section{TESTING COMPARISON BETWEEN 3D DATA OBTAINED BY GOOGLE EARTH AND THOSE OBTAINED BY SURVEY WITH DRONE AUSILY}

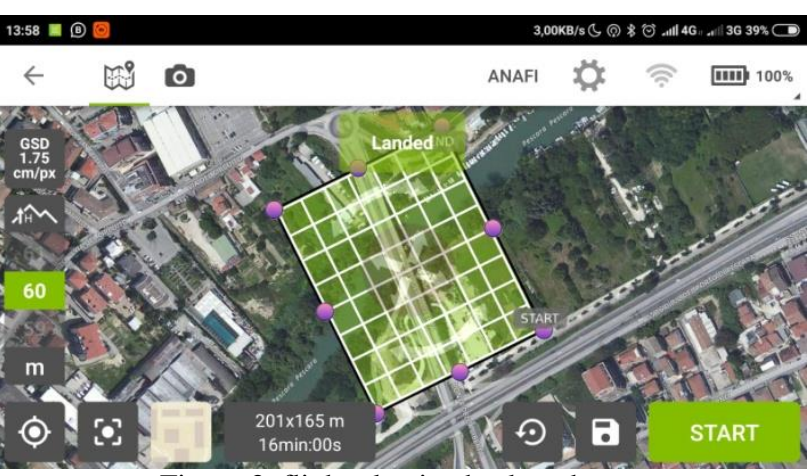

Figure 3: flight plan in checkered area.

Despite the good level of detail obtained by the digital model being returned from the open source data granted by Google, it was decided to verify the threshold of error resulting from a virtual cloning operation so articulated through a specific project of image based survey, with RPAS support, on the sample spaces of the case study, specifically a central area along the river basin of Pescara consisting of rather heterogeneous characteristics with spontaneous vegetation and built-up areas, with the presence of a bridge that connects the two shores, some boathouses and abandoned spaces. In the case study, carried out with an image-based system, the small Parrot Anafi drone was used, equipped with 4 rotors, with integrated $21 \mathrm{mpx}$ Gimbal. The take-off weight of this drone is about $400 \mathrm{~g}$ with a flight autonomy of 25 minutes in normal conditions with no wind and with a regular flight (Figure 3).

As in the classic aero-photogrammetry, where the cameras on board the aircraft are mounted on special stabilizers, also for the drones are used anti-vibration balancing and suspension systems, called in terms of Gimbal, precisely stabilizers. Obviously, they are chosen according to the type of camera used and must be calibrated and set by the operator. The stabilizers are able to control and in case correct the inclination of the camera in the three axes $\mathrm{x}, \mathrm{y}, \mathrm{z}$, in this way the same angle of inclination will be guaranteed for the whole survey regardless of the oscillations of the drone itself. Special rubber pads also allow you to dampen all the oscillations and vibrations of high frequency that are generated during flight operations (Palestini and Basso, 2017). Through a remote control the ground operator is able to control and manage the flight of the sapr and always through the same device we can manage the camera stabilizer so as to allow, if necessary, to vary during the flight the inclination of the camera itself. Another function that is possible through a remote control is to take pictures and start or stop the video recording. The data acquisition from the Google Earth support has followed as much as possible the same flight settings of the real drone, such as the height, about 90 meters from the ground, and the precise inclination in degrees of the optical cones of the cameras. In addition, the images captured by the virtual tour were recalibrated based on the actual conformation of the lens used in the drone survey. Despite the apparent differences, in essence acquire photographic images using the methodology structure for motion follows the same principle of homology and formulas that allow us to determine the height of the flight itself, the frequency of shooting of photos (taking base) and the distance of the various steps, depending on the restitution scale. In the flights effected with drone, in analogy to the air flights, beyond to establish a base, it is necessary to establish also a distance between the various data progressive crawlings, so to guarantee that there is also a lateral covering $\varepsilon$ (overside) that generally varies from $10 \%$ to $20 \%$. The calculation of the base of shooting and the distance between centres (overlap and overside) allow us to carry out a survey without the risk of having some zones not adequately acquired (De Luca, 2011).

Another fundamental datum to take into consideration in an image based survey is the GSD (Ground Sample Distance), that is the sampling distance on the ground, which indicates the degree of resolution of our survey, expresses the distance between the centre of two consecutive pixels expressed in units of territorial measure, or in other words expresses the amount of land represented by 1 pixel in the image, is measured in $\mathrm{m} /$ pixels or $\mathrm{cm} /$ pixels that is determined through a proportion between similar triangles as shown in the diagram (1d):

$\mathrm{d}: \mathrm{D}(\mathrm{GSD})=\mathrm{f}: \mathrm{H}$

where:

$\mathrm{d}=$ pixel size

$\mathrm{f}=$ focal length

$\mathrm{H}=$ gripping distance

$\mathrm{D}(\mathrm{GSD})=$ ground sampling distance

So:

$\mathrm{D}(\mathrm{GSD})=(\mathrm{d} \mathrm{H}) / \mathrm{f}$

Generally the GSD value for a good relief should be less than $5 \mathrm{~cm} /$ pixel (Cundari, 1984).

The result of the comparison between the model obtained from images taken directly from the desktop, derived from the Google platform, and the virtual model developed on site through UAV system, with the same methods of digital acquisition, has certainly revealed a real error but undoubtedly extremely contained in the light of the type of experimental operation carried out. To strengthen the verification, a further check was provided by the cartographic data from which the trust points and the IGM quotas were assimilated, which serve as a control to measure the detection error inherent in the described return methodologies, in order to have a set of data on the average error, information that is then used as corrective parameters to recalibrate the model and geo-reference it.

The permissible error is related to centimetre and is considered on the flight altitude and the actual resolution of the optics used. We have an interesting comparison between what are the absolute points and the relative deviation or error that results from cloud returned by drone and cloud returned by Google. In fact, the first difference that comes out of the models is the measurement in scale. The drone takes photographs with both position information and technical specifications of the optics, thus allowing the software to create a model already plausibly to 
scale. Otherwise it happens on the Earth model, as the source is always images, but without any information of any kind. The software generates a proportionate model but to which a measure must be applied in order to scale the model. The resulting error is totally related to the resolution of the images, the less the detail, the more the difference will be. To experimentally evaluate this error we have added points taken with precision GPS inside the clouds created, generating markers, as absolute points on which to bind the model. Essentially, by detecting and positioning at least 4 real points and materializing them within the generated cloud, we force the program to make the same virtualized points coincide with those taken materially. This operation will generate an error due to the corrective adaptation that the software will apply to the model in order to make it pass through the markers that we have detected and established, error appreciable from the following images and reports. As you can see the drone model is below the average $5 \mathrm{~cm}$ error, and this makes us appreciate the fidelity with which the model was generated to scale. Note, however, the enormous difference in positioning of the model in the $\mathrm{X}, \mathrm{Y}$ and especially $\mathrm{Z}$ axes.

This gives us two important information, the first is that the model itself is very close to the real scale because the GPS information inside the drone is truthful enough to allow the software to probably sample the point cloud, but at the same time, being a low-cost commercial GPS, it clearly suffers the metric error of positioning in space.

For the Google Earth model, on the other hand, the subject is different. Not having GPS information results to be out of scale and above all and randomly positioned in space. Nevertheless, the model is correctly and proportionally processed, so having a certain measure, it can be scaled correctly. This measurement is taken directly on the spot or with the Google Earth ruler, and this leads to interesting implications about the reliability of the model and the potential for processing. The latitude and longitude coordinates are very close to those taken with precision instruments (Figure 4-5). The greatest lack of information is found in relation to the elevation, since Earth defines on the $\mathrm{Z}$ axis the measurements of heights at scans of one meter at a time, generating a positioning inaccuracy. This data is interesting because this measurement is closer to the actual altitude than that of the drone, which also applies its shooting height to the real altitude, since it cannot be precisely defined as it is subject to wind and constant flight corrections. A proportional similarity of the data taken from the physical drone model and from the Google Earth model can therefore be found: the error with respect to the real measurements is only due to the size of the pixels that give the detail (Figure 6). It is useful to position the Earth model, already equipped with coordinates that can be taken directly from both the Google Earth program and the Google Maps platform. The data of these coordinates are very close to those taken with precision instruments, the error is at the centimeter for the $\mathrm{X}$ and $\mathrm{Y}$, while maximum per meter for the $\mathrm{Z}$ axis. It is therefore possible to create a low cost model essentially combining the definition by drone and the centimeter accuracy of the Google coordinates, obtaining a model verified in scale with minimal error and at the same time being able to position it in space almost absolutely, in the $\mathrm{X}$ and $\mathrm{Y}$ axis, with an error of up to one meter (in the current version of the program) in the $\mathrm{Z}$ axis. This opens multiple scenarios regarding the acquisition to reduced budget in whatever type of context is in relation to the restitution in $2 \mathrm{~d} / 3 \mathrm{~d}$, both in function of a project and analytical use.
In specific, we analyze the pipeline of the case study.

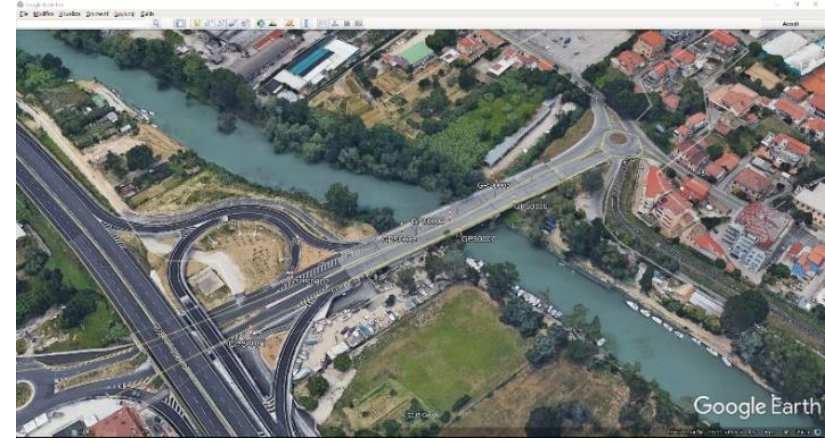

Figure 4: acquisition of 8 GPS certain points.

We carried out the survey with the drone through software that allowed us to carry out in an automated way a flight plan, of grid type, to acquire the necessary photos.

On the spot we acquired 8 points with precision GPS, useful to have an absolute and certain basis of the measurements and positions of the elements that we later have bound.

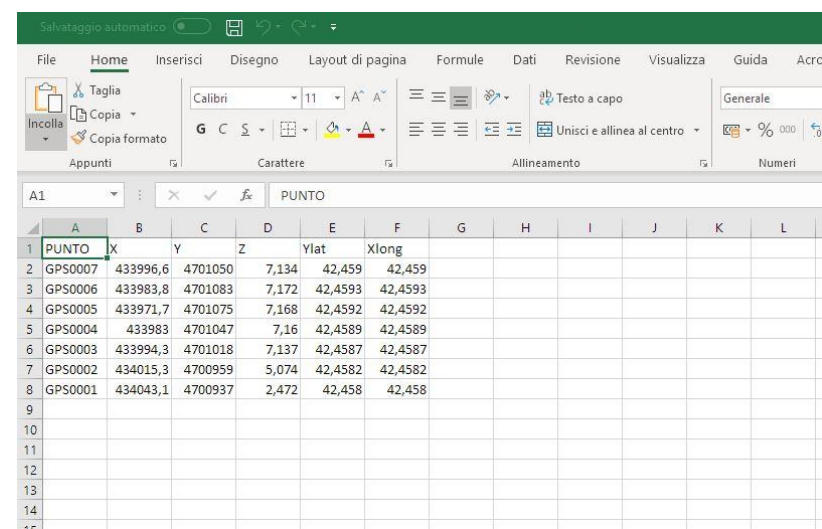

Figure 5: acquisition of 8 GPS certain points, coordinated in the workspace, necessary to have absolute measurements for comparison.

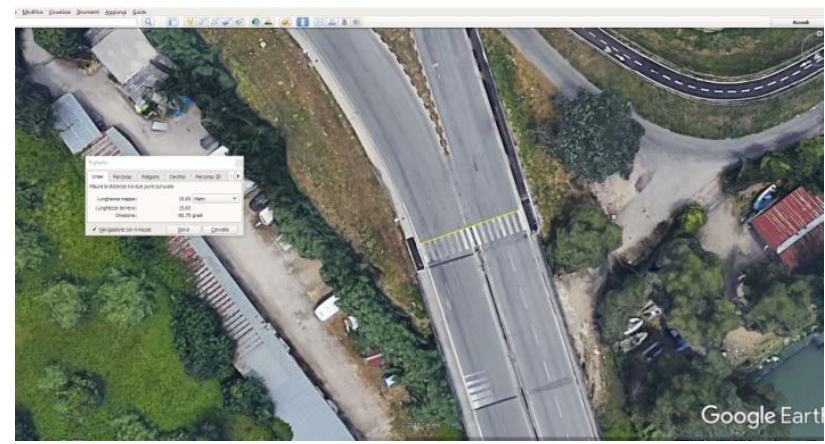

Figure 6: the measurement acquired by Google Earth is 15.6 metres.

We then proceeded by acquiring the grid closest to our study area from the Military Geographic Institute to operate correctly the conversions of the coordinates taken and to have available data already verified previously (Figure 7). 


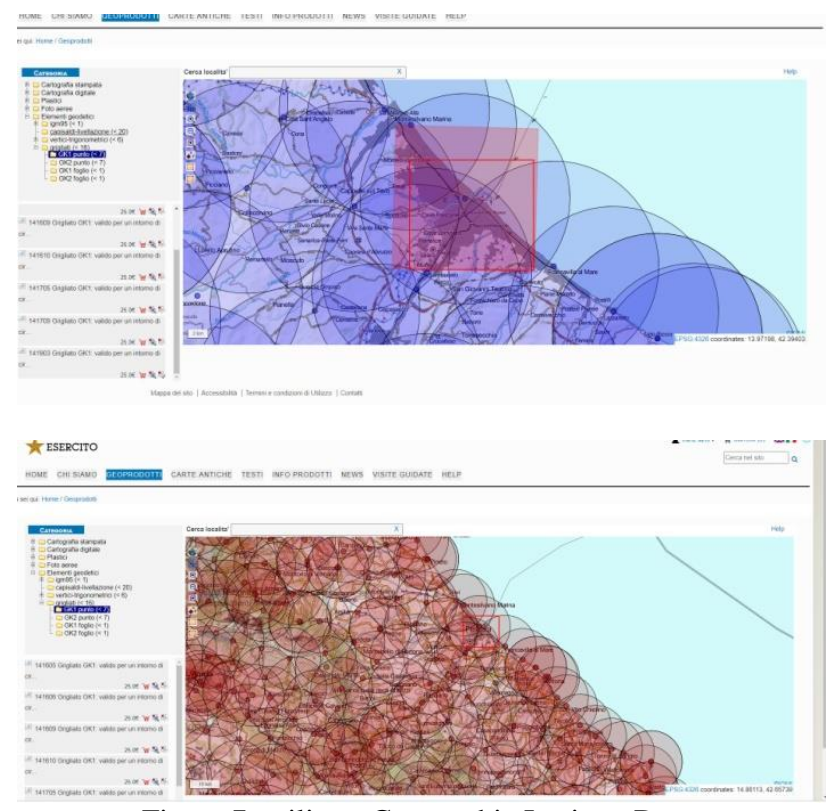

Figure 7: military Geographic Institute Date.

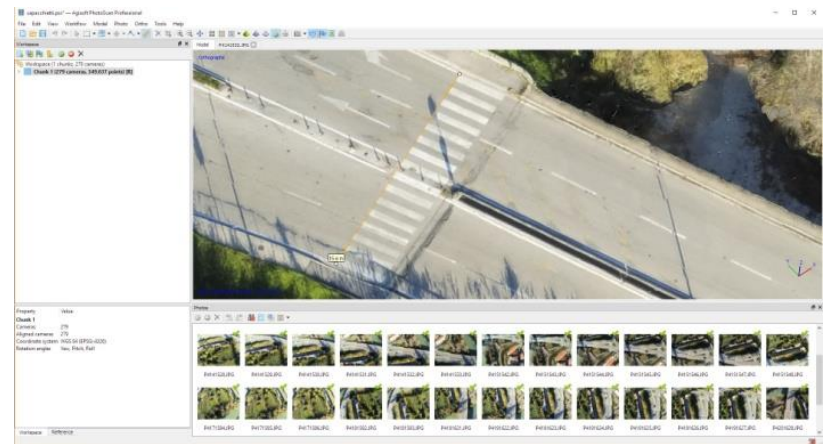

Figure 8: the measurement taken by the drone model without applying the GPS point correction is 15.6 meters.

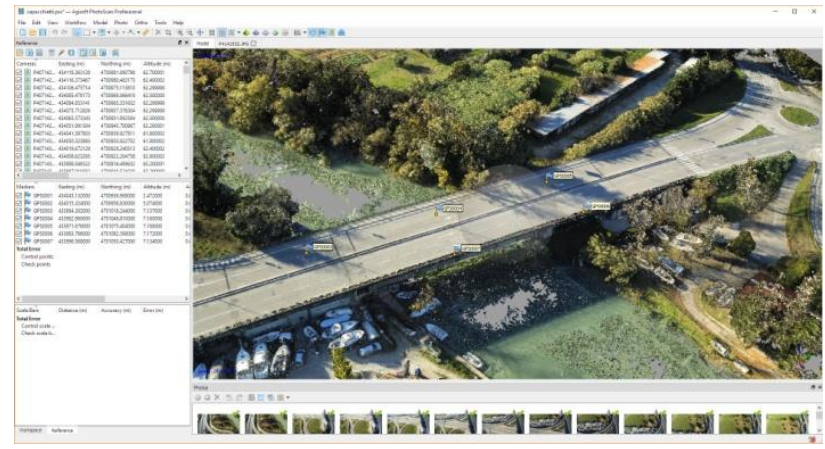

Figure 9: the 5 point GPS mismatch materials taken on site with the model directly generated by drone.

The model elaborated by the drone flight has given interesting results, being consistent with the measures taken on the spot and consistent with the measures taken by Google Earth.

From the sequence of the images it is possible to visualize the different phases of work, comparing the waste produced with respect to the correct model according to the points detected with GPS. As you can see, the measurement is always 15.6 meters (Figure 8), while what changes is only its obviously more precise positioning in the three-dimensional georeferenced space (Figure 9-10).

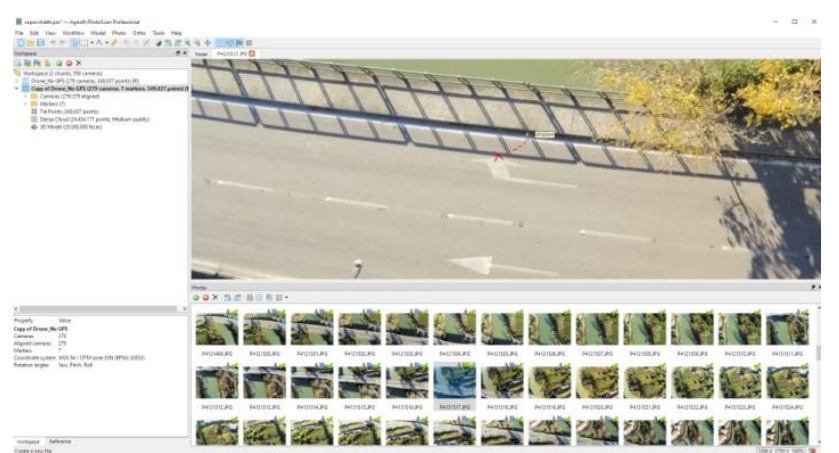

Figure 10: a detailed view of how different the point taken with GPS and the result of the drone cloud differ.

\section{Ground Control Points}

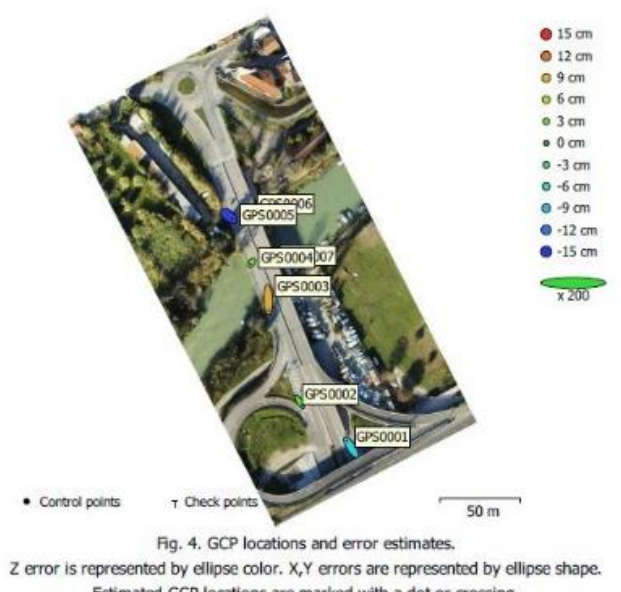

Estimated GCP locations are marked with a dot or crossing.

$$
\begin{array}{|l|l|l|l|l|l|}
\hline \text { Count } & \text { X error } \mathbf{( c m )} & \text { Y error }(\mathbf{c m}) & \text { Z error }(\mathbf{c m}) & \text { XY error (cm) } & \text { Total (cm) } \\
\hline 7 & 1.56811 & 3.7269 & 8.79127 & 4.04336 & 9.67653 \\
\hline \multicolumn{5}{|c|}{\text { Table 4. Control points RMSE. }} \\
\text { X- Easting, Y - Northing, Z - Altutude. }
\end{array}
$$

Figure 11: ground verification points. GCP location and error estimates in drone survey.

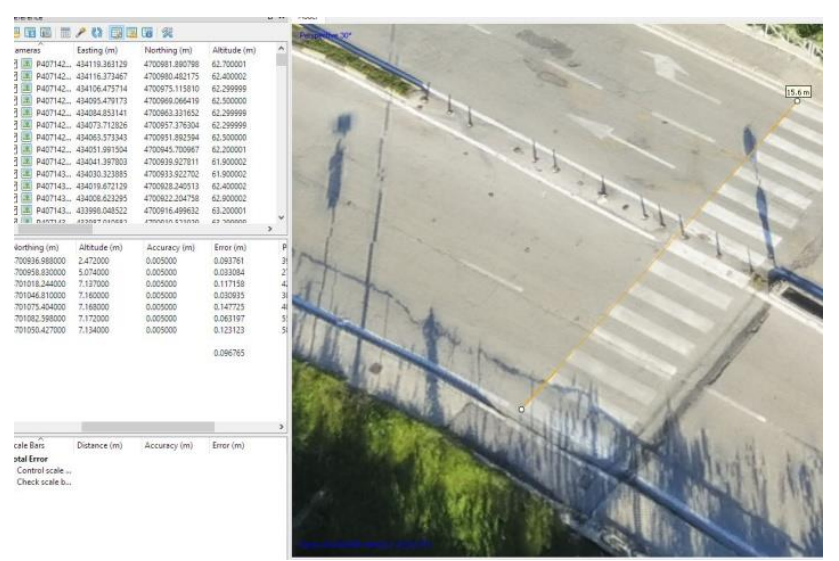

Figure 12: the table allows us to define the error accumulated in the area with respect to the verification points. 


\section{Camera Locations}

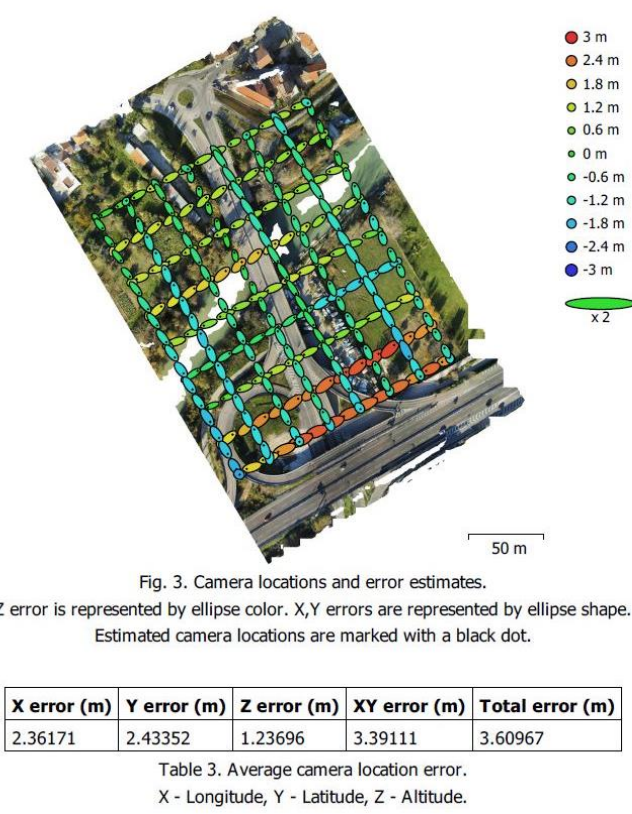

Figure 13: auto-correction on the camera that the program applies to obtain the model related to reality.

\section{Camera Locations}

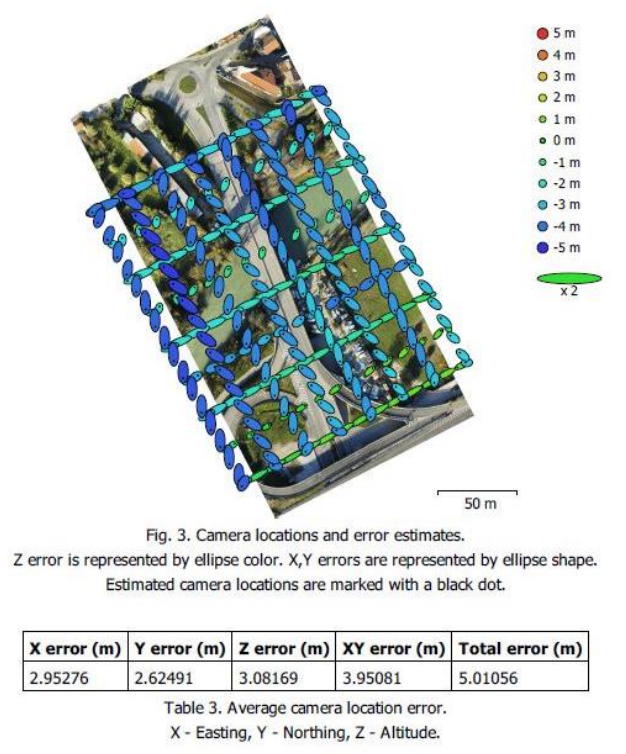

Figure 14: auto-correction of the rooms with the additional help of GPS points in order to have a valid camera alignment.

The model generated by the images taken from Google Earth obviously suffers limitations such as loss of resolution, no georeferencing and obviously no exif focal data. Despite this, the software processes the point cloud from the sequence of 60 photos taken manually by simulating the drone grid (Figure1314).

The interesting result reveals that it is possible to apply the scale factor and the GPS coordinates taken physically on the generated cloud (Figure15). Once you have applied the GPS coordinates you can see the generated error of 0,40 meters. From the table, the accumulated error is due to the uncertainty in the ratio between the pixels and the GPS markers applied.

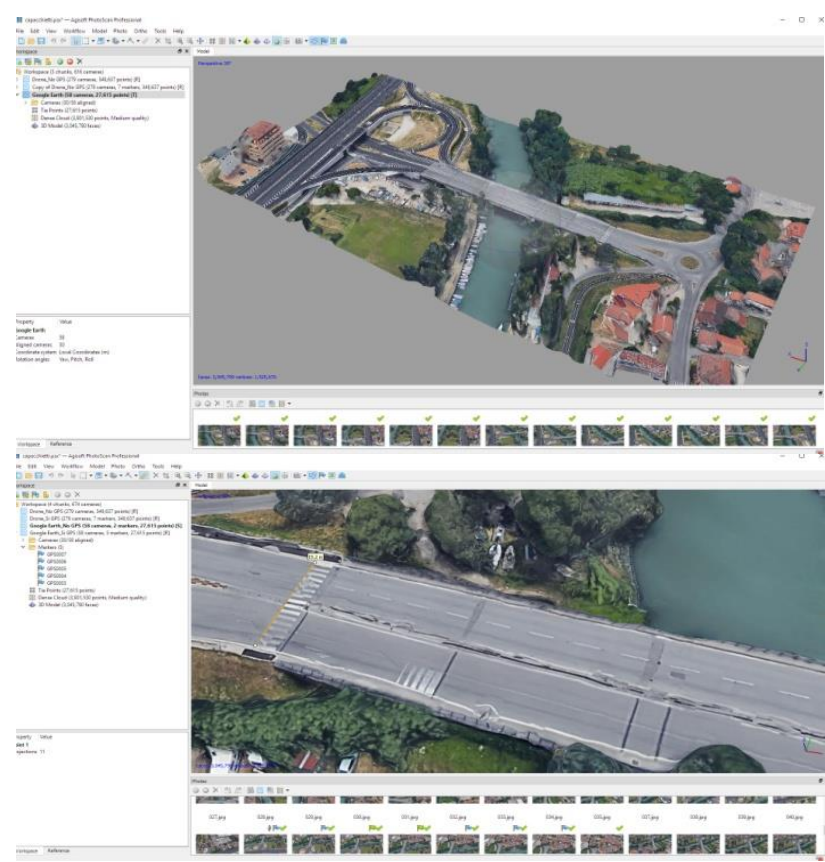

Figure 15: the scale factor and the GPS coordinates taken physically on site on Google model.

\section{Ground Control Points}

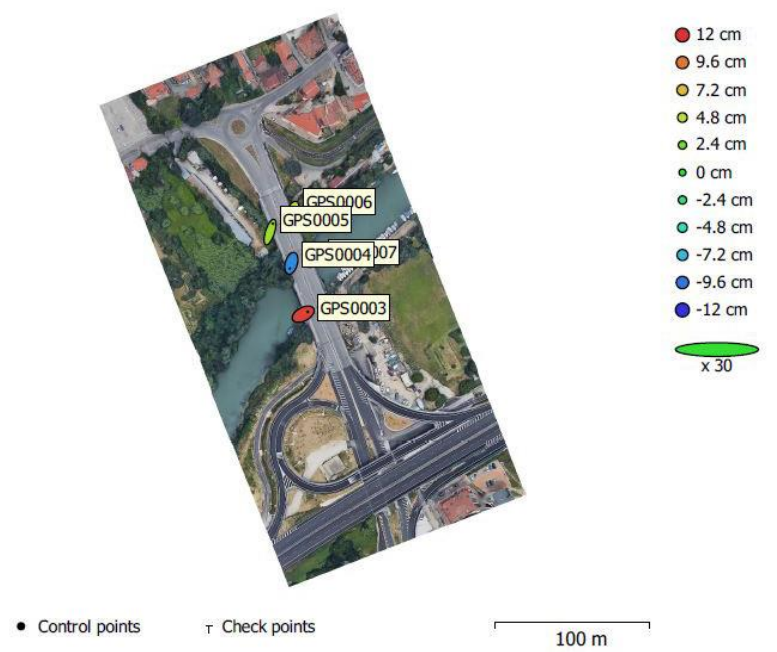

Fig. 3. GCP locations and error estimates.

$Z$ error is represented by ellipse color. $X, Y$ errors are represented by ellipse shape. Estimated GCP locations are marked with a dot or crossing.

\begin{tabular}{|l|l|l|l|l|l|}
\hline Count & X error $(\mathbf{c m})$ & Y error $(\mathbf{c m})$ & Z error $(\mathbf{c m})$ & XY error $(\mathbf{c m})$ & Total $(\mathbf{c m})$ \\
\hline $\mathbf{5}$ & 13.962 & 21.8432 & 7.78168 & 25.9242 & 27.0669 \\
\hline \multicolumn{6}{c}{ Table 3. Control points RMSE. }
\end{tabular}
$X$ - Easting, $Y$ - Northing, Z - Altitude.

Figure 16: ground verification points. GCP location and error estimates in Google survey.

The results show that having certain measurements on the ground it is possible to obtain reliable models through the photogrammetric technique (Figure16). In short, the choice is linked to the purpose of the survey, the material points can be taken either with precision instrumentation, taking into account the accuracy and territorial scale of the project to be 
implemented, or by materializing the points directly in virtual from Google Earth combining them with a drone-shot.

\section{DATA PROCESSING FOR TRANSFERRING TO A VIRTUAL PLATFORM.}

The preventive operation of dividing the study band into quadrants, which can be overlapped by a few dozen metres in order to generate an optimal stitching of a compact model during the compositing phase, has allowed the formulation of a precise digital reconfiguration project following an orderly executive workflow, from which two textured models have been obtained for each single quadrant analysed: a lowpoly asset, therefore with low polygonal density, obtained exclusively by exploiting the various depth maps obtained from $2 \mathrm{~d}$ map data from photogrammetric photoplan, Diffuse-color maps, Bump, Normal and Displacement, and a high definition model, completely $3 \mathrm{~d}$, always the result of the previous phases of photo modelling, with the integration of data from three-dimensional satellite maps and elevation and proportional information from the Dem-open source mapping (Figure 17).

The creation of two different models, coinciding of the same environment, aimed primarily at an explanatory verification of the criticality and connotative characters on the various study blocks, can be considered functional to the eventual possibility to transfer of the same scenario reconfigured on an interactive platform explorable in real time, in compatibility with a project aimed at territorial control, methodology that necessarily requires the implementation of different levels of detail, in programming language "LOD" (Level of Detail), consisting of multiple $3 \mathrm{~d}$ models identifying the same section of territory, the polygonal density progressively increasing, so as to be able to manage optimally, by the hardware, considerable polygonal volumes by replacing the low-poly model with the high-poly, depending on the distance from the virtual camera. This methodology allows the insertion of separate additional data in various layers of detail, in order to enrich the virtual exploration experience, allowing greater versatility and a more accessible understanding of the model reproduced and the actual morphology of the real place of reference (Santagati et al., 2013).

Very frequently after the phases of photo modelling artifacts or definition errors may occur due to the complexity of the reconfigured scenario and the digital disturbance resulting from the irregularities of the spontaneous vegetation identifying the river basins, which in the auto modelling procedures generates gaps and imperfections that make the $3 \mathrm{~d}$ model unsuitable for direct export to rendering real time platforms such as Unreal 4. Despite the fact that Agisoft product already contains a series of correction algorithms, it was preferred to proceed, to solve these
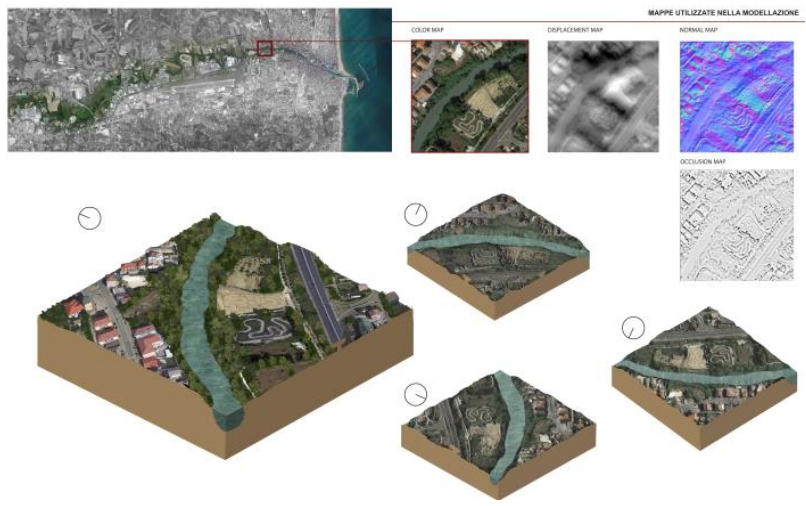

Figure 17: low-poly model obtained from photoplan and satellite data.

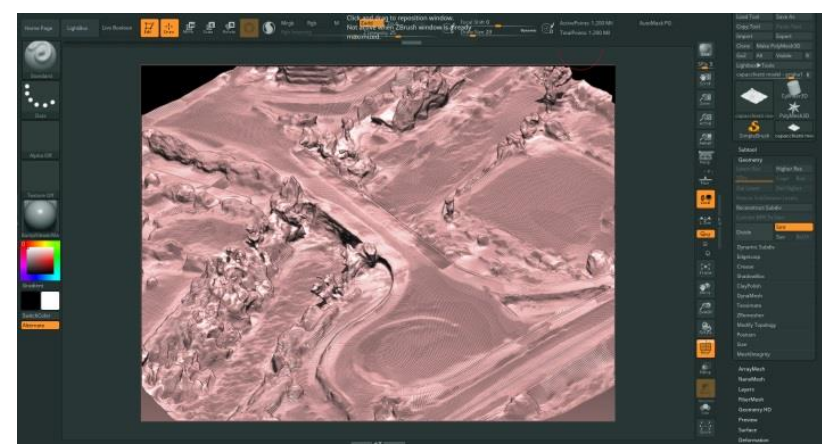

Figure 18: 3d photogrammetric model optimized in Zbrush and retopologized from triangular to poly-quod.

meshing problems, exporting the model and working with the sculpting software, Pixologic Zbrush according to the desired visual quality and in relation to the scale of the threedimensional asset to be displayed. To follow this procedure it is necessary to intervene on the topology of the $3 \mathrm{~d}$ model and on its optimization (Figure 18).

Through some exceptional features of Zbrush, such as the ZRemesher, fundamental in the architectural field for model correction, intelligent retopology, creation of detail and modification on the UV mapping compatible with the triangular model with high polygonal density, it is possible to optimize the three-dimensional assets in function of a full compatibility for the next phase of import on the current virtual platforms of real time rendering. The possible implementation of further detailed parametric models for the animated rendering of biological elements, such as parametric vegetation or BPR procedural shaders to identify the river water, allow to obtain in Unreal Engine good visual effects of photorealism, offering infinite possibilities for VR interaction and an easier exploration of complex three-dimensional models relating to reconfigured macro-areas, facilitating their use in professional environments with the most varied connotations, from 'Architecture to museum education, from archival studies to territorial and geological analysis, studies on tree varieties and morphological metamorphosis of water basins.

A good 3d representation of vegetation is important for the definition of a credible urban model, both for its essential identification footprint, and for the particular roles it plays in reality, and therefore assumes an importance related to graphic and visual simulations, (temperature, pollution, noise, water, human perception, etc..), both for its impact in the acquisition of data for urban modelling (road morphology, occlusion of greenery, reference point for the recording of point clouds). However, greenery is difficult to reconstruct due to a number of interlinked factors, such as complexity, multi-scalarity of models and volume variability. Since the definition of tree species shows an irregular but hierarchical nature, Unreal Engine's proprietary procedural modelling methods seem to be the most suitable, also because they provide additional animated effects, such as the contribution of wind between the foliage and the use of sub-surface scattering materials that excellently simulate the effect of light as it passes through the foliage (Figure 19). The propagation of vegetation could thus depend, at an algorithmic/procedural level, on the physical morphology of the site proposed in the digital model, following automated procedures that in any case follow the actual conformation of the soil.

In an optimal 3D reconstruction, the green is important in contributing realism to the model and because it geometrically provides a diffuse organic form, as opposed to the regular and compact form of buildings or roads. 
On an urban scale, a specific modelling strategy for trees may be needed, as any use of tree models must introduce reasonable hardware requirements. To this end, one solution is to have different models for the same tree with different levels of detail, which could also be the case for buildings in the analysis area, which still follow a smaller variety of polygonal models. Individual tree modelling is thus an interesting objective to pursue. A less detailed model can be rendered when the tree is away from the viewer, while the more precise model is shown when the tree is near the view camera, producing different levels of detail for each tree model. When trees are grouped, you can also rely on custom methods to allow a realistic and quick view of the highly optimized elements (Magallanes Guijón et al., 2018). In anticipation of the involvement of external programs to Unreal Engine for the definition of green, we can use as parametric modellers software such as Plant Factory, Speed Tree, EvyGen and Forester, helping to build in a simple and intuitive way both existing tree species, which can develop along a real path of growth (growing up), or plant species created for better optimization for hardware use that make the visualization of complex urban models more fluid. Then, auto distribution algorithms that use tools such as vector painting are implemented, which literally allows you to paint trees in the areas we prefer, or automatic systems that decide the tree density and type based on physical factors and elevation of the land on which the urban model is distributed.

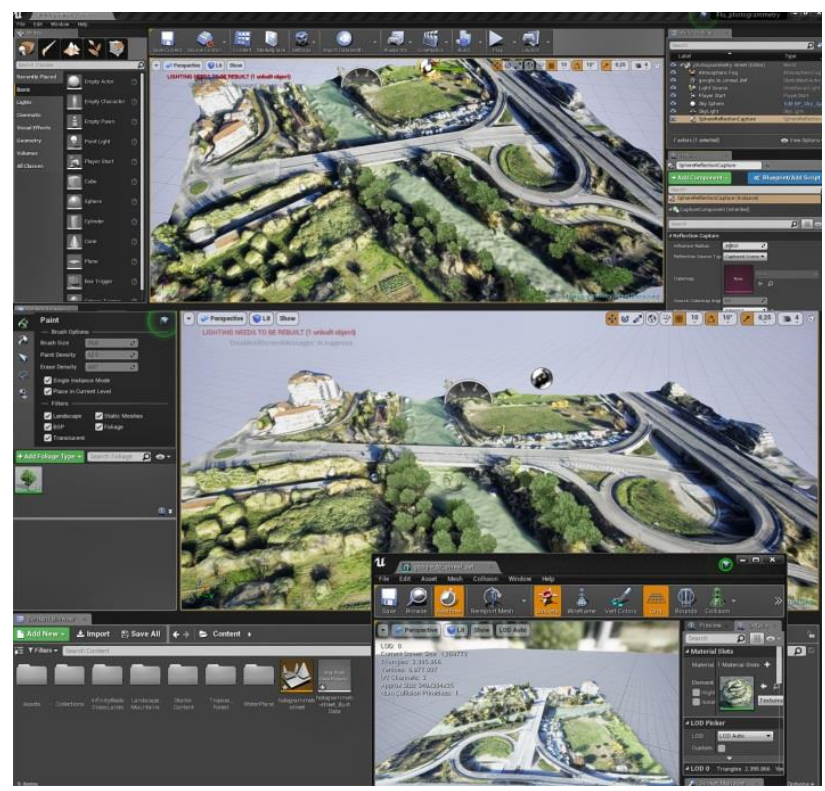

Figure 19: optimized model transfer on Unreal Engine and addition of parametric vegetation.

\section{CONCLUSIONS}

In short, the contribution through the described methodology offers study models suitable to reconstruct and explore threedimensionally complex territorial situations through integrated detection systems and software capable of offering endless possibilities of interaction VR applicable to the study of the territory, orographic and geological conditions (Sharkawi and Abdul-Rahaman, 2008). Specifically, the focus has been on the analytical exploration of river environments in which different and heterogeneous aspects related to tree varieties, to cyclical metamorphoses of water basins subject to seasonal changes, which require general and thematic models of study, are added. In conclusion, without prejudice to the fact that the choice of survey methods remains linked to the aims and objectives to be achieved, in which the margins and the acceptability of errors come into play, the experimentation has offered excellent results considering the vast territorial extension and the scale ratios on which to operate, as well as the versatility and the possible integration obtained with low-cost systems that have allowed a rapid and effective exploration of the environmental complexities inherent in river basins (Napolitano et al., 2018).

\section{ACKNOWLEDGEMENTS}

The authors would like to thank in particular the people involved in the acquisition of data and in the campaign on site including Franz Lami for the filming and processing by drone and for the processing of image-based data and the geologist Matteo Mirabilio for the acquisition of GPS points functional to the verification phases.

\section{REFERENCES}

Cummings, J., and Bailenson, J., 2016. How immersive is enough? A meta-analysis of the effect of immersive technology on user presence. Media Psychology, 19(2), pp. 272-309. http://dx.doi.org/10.1080/15213269.2015.1015740

Cundari, C., 1984. Fotogrammetria Architettonica. Bologna, Italia: Kappa Ed

De Luca, L., 2011. La Fotomodellazione Architettonica.Palermo,Italia: Flaccovio D. Editore.

Magallanes Guijón, G., Hruby, F., Ressl, R., Aguilar Sierra, V., de la Borbolla del Valle, G. and Rodríguez Paz, O., 2018. Modelling of collective movement in immersive environments. In: International Archives of the Photogrammetry, Remote Sensing and Spatial Information Sciences, XLII-4, 397-402.

Napolitano, R.K., Scherer, G. and Glisic, B., 2018: Virtual tours and informational modeling for conservation of cultural heritage sites, Journal of Cultural Heritage, 29, 123-129.

Palestini, C., Basso, A., 2017: Geomatics as a survey tool to document and enhance the cultural and landscaped heritage of the monumental complexes in the mountains of Abruzzo, In: International Archives of the Photogrammetry, Remote Sensing and Spatial Information Sciences - ISPRS Archives, XLII-5/W1, 373-380.

Santagati, C., Inzerillo, L., Di Paola, F., 2013. Image-based modeling techniques for architectural heritage $3 \mathrm{~d}$ digitalization: limits and potentialities, in International Archives of the Photogrammetry, Remote Sensing and Spatial Information Sciences, Vol. XL-5/W2, 20. pp. 550-560.

Sharkawi, M.U., Abdul-Rahaman, A., 2008. 3D Navigation system for virtual reality based on $3 \mathrm{~d}$ game engine. In: International Archives of the Photogrammetry, Remote Sensing and Spatial Information Sciences. Vol. XXXVII.

Soler, F., Melero, F.J., Luzón, M.V., 2017 : A complete 3D information system for Cultural Heritage documentation, Journal of Cultural Heritage, 23, 49-57. 